\title{
EVALUATION OF A PATIENT REFERRAL CONTACT TRACING PROGRAMME FOR HEPATITIS B AND C VIRUS INFECTION IN DRUG INJECTORS
}

\author{
D D Brewer (interscientific@yahoo.com)1, H Hagan² \\ 1. Interdisciplinary Scientific Research, Seattle, United States \\ 2. National Development and Research Institutes, Inc., New York City, United States
}

\begin{abstract}
Effective contact tracing for hepatitis $B$ virus (HBV) and hepatitis $C$ virus (HCV) infection could enhance disease control, especially in populations with low HBV vaccination rates and high prevalence of untreated HCV infection. We evaluated a low-cost approach to HBV/ HCV contact tracing in injection drug users (IDUs). Index cases $(n=26)$ were IDUs who seroconverted to HBV and/or HCV during a prospective cohort study in Seattle. Interviewers elicited index cases' recent injection partners and administered recall cues and other techniques to boost recall. Index cases received vouchers for free hepatitis testing, which they were to give to locatable partners. Persons redeeming vouchers also received small monetary incentives. Most (26/40) seroconverters participated in the paid contact interviews. Index cases reported many partners (mean=17), and in the aggregate, index cases indicated they could refer more than one third of their elicited partners for testing. Overall, only 17 persons were ultimately referred and just eight of these were confirmed as partners sought for referral. The supplementary elicitation techniques, and especially the recall cues, increased reporting of injection partners substantially. The injection network constructed from reported partnerships was mostly connected and cyclic. Successful contact tracing in IDUs likely requires active involvement by public health staff to locate and notify exposed injection partners.
\end{abstract}

\section{Introduction}

The two basic approaches to contact tracing for infectious disease are provider referral and patient referral $[1,2]$. In provider referral, health workers elicit infected persons' contacts, obtain identifying and locating information on those contacts, and then locate them to notify them of their exposure and provide counselling, testing, treatment and/or vaccination. In patient referral, infected persons are instructed by health workers to notify their contacts and refer them for testing, treatment and/or vaccination.

Contact tracing for hepatitis $\mathrm{B}$ and $\mathrm{C}$ viruses (HBV and $\mathrm{HCV}$ ) in most communities defaults to patient referral or even no contact tracing at all. In some communities, contact tracing may also be initiated by public health staff in response to a case report or a request from a clinician or patient, but such efforts may be quite limited due to the extremely low rate of $\mathrm{HBV} / \mathrm{HCV}$ case reporting, particularly for injection drug users (IDUs) [3,4]. Health departments in a few communities, however, have implemented routine, proactive HBV control programmes that include tracing index cases' sexual and household contacts $[5,6]$. To our knowledge, no routine contact tracing programmes have been described in the literature for HBV in IDUs or for HCV in any population.

Nevertheless, there are potential disease control benefits of effective HBV/HCV contact tracing programmes for IDUs. IDUs have low rates of HBV vaccination (in recent studies, $4-22 \%$ of IDUs without active or past infection had been vaccinated [7-9]) and contact tracing offers an opportunity to vaccinate susceptibles who have had risky contact with infected persons. Also, through HCV contact tracing, many HCV-infected IDUs can be identified and evaluated for treatment of their infections. IDUs treated for HCV infection, including those who continue to inject, show effective sustained virologic responses comparable to patients without a history of illicit drug injection, even over long post-treatment follow-up periods [10].

In this article, we report an evaluation of a patient referral contact tracing programme for HBV and HCV infection in IDUs. We also describe the impact of supplementary interviewing techniques for eliciting contacts and assess epidemiologically relevant aspects of the injection network ascertained through the programme.

\section{Methods}

\section{Participants}

Participants were IDUs who seroconverted to HBV or HCV during a prospective cohort study of incident HCV infection conducted in Seattle, United States (US), between 2004 and 2006. IDUs were recruited into the cohort study from the county jail, two needle exchange programmes, street locations where IDUs were present, and by referral from another research study and from eligible participants. Individuals who had injected drugs at least once in the previous six months, spoke English, were 15 years of age or older and tested negative for HCV antibody were eligible for the cohort study. Cohort study participants were scheduled for HBV and HCV antibody testing and counselling every six months during the study period. Sera were screened for HCV antibody (anti-HCV) with a third generation enzyme immunoassay (Abbott Laboratories, Chicago). Sera were screened for hepatitis B core antibody (anti-HBc) using an enzyme immunoassay (Abbott Laboratories, Chicago). Anti-HBcseronegative participants, some of whom may have been previously 
vaccinated, were referred to free HBV vaccination services near the study office. (None of the study staff were licensed nursing or medical providers and consequently were legally prohibited from vaccinating participants.) Seroconversion was determined by the appearance of anti-HCV or anti-HBc in a previously seronegative individual.

A total of 211 IDUs completed at least one of the follow-up assessments. During the course of the cohort study, 36 participants seroconverted to HBV and/or HCV, and 23 (64\%) agreed to participate in the contact tracing study, which was formally separate from the cohort study. (Unfortunately, we did not collect data on reasons for not participating in the contact tracing study.) All seroconverters were referred to a hepatitis clinic for free medical evaluation. Four additional IDUs with prevalent HCV infection at baseline were also invited, inadvertently, to participate in the contact tracing study; three of them agreed to participate. For ease of presentation, we group these additional cases with the prospectively identified seroconverters. Thus, the overall participation rate in the contact tracing study was 65\% (26/40). Participants in the contact tracing study received USD 20 for their participation, and all provided written informed consent. The University of Washington Human Subjects Review Committee approved the study.

\section{Interviewing procedures}

Four trained study staff served as interviewers for this study (including one who had served as an interviewer in a prior HCV contact tracing study [11]). At the beginning of the interview, interviewers explained to index cases the purposes of contact tracing and how the interview related to those goals. Interviewers then asked index cases to recall their injection partners during the 12 months preceding the interview, a period which encompassed most or all of the time after index cases had seroconverted. Injection partners were defined as persons who had injected drugs together with an index case, regardless of whether they had shared needles and including persons who injected the index case and persons whom the index case injected.

Interviewers began eliciting partners by asking index cases to list their partners freely. When index cases indicated they did not have or could not recall any more partners, interviewers prompted non-specifically (e.g. "who else did you inject drugs with ...?"). Interviewers prompted in this way until the index case insisted he or she could recall no additional partners. Next, interviewers slowly read the list of elicited partners back to the index case to ensure the partners were correctly recorded, and then prompted non-specifically again.

At this point, interviewers briefly explained to index cases that our past research showed that people often forget some of their partners. Interviewers then administered location and network recall cues to elicit additional injection partners [12-14]. We used the same 17 locally-relevant and empirically-derived location cues for enhancing recall of injection partners that we had used previously with Seattle IDUs $[13,14]$. For the location cues, interviewers asked index cases to think of all the people they had met or injected with at a particular place (e.g. a motel) during the recall period, and to list any additional partners if they had forgotten to mention them earlier. For the network cues, interviewers read back the list of partners elicited so far in the interview. For each partner, the interviewer asked the index case to think of other people who interact with or know that partner and to list those with whom the index case had injected during the recall period but whom they had forgotten to mention earlier.

After elicitation, index cases indicated those partners whom they knew how to locate and those whom they planned to refer to the contact tracing study. Interviewers collected first names or street nicknames for all elicited partners.

\section{Referral procedures}

Index cases received numbered vouchers to give to the partners whom they agreed to refer to the study for testing. Each voucher indicated that it could be redeemed for USD 5-15 for participation in a confidential study on drug use and health, and listed a telephone number for scheduling an appointment. Interviewers coached index cases how to refer their partners. Index cases were to emphasise that hepatitis was highly prevalent in IDUs and that study participation involved free hepatitis testing, and they were also to highlight the benefits of such testing. Index cases were not instructed to report their seroconversions to their partners or notify them of specific potential exposure to HBV and/or HCV.

When referred persons scheduled their appointments by telephone or came to the study site to redeem their vouchers, interviewers determined whether they matched any of the partners whom the corresponding index cases had agreed to refer. Matching was based on the voucher redeemer's name, voucher number and name of the person the redeemer said they had been referred by. Referred persons who did not appear to match the sought partners were still eligible to participate. Voucher numbers were not required for participation, if the index case accompanied the referred person to the study site. Each referred person was paid USD 5 for redeeming the voucher and was then offered free counselling and antibody testing for HBV and HCV. Those who received counselling and testing were paid an additional USD 10. Persons testing anti$\mathrm{HBc}$-negative were referred for free HBV vaccination and those testing positive for HBV or HCV were referred to the hepatitis clinic for free medical evaluation.

\section{Statistical analysis}

We computed descriptive univariate statistics on index cases' characteristics, contact tracing outcomes, and the number of partners recalled at different stages in the interview, as well as proportional increases in the number elicited during different stages. To assess the representativeness of participating seroconverters, we compared them with seroconverters who did not participate in the contact tracing study in terms of demographics, drug use and injection risk behaviours, using appropriate measures and tests of association. We also calculated Pearson correlations for selected predictors of the number of additional partners elicited by the supplementary techniques. In addition, we computed Pearson correlations for each index case between whether a partner was elicited before or by the recall cues and whether a partner was locatable. We summarised these coefficients with the mean correlation weighted by the number of partners elicited and the associated cumulative Z score [15]. Finally, we produced a graph of the injection network with a spring embedder algorithm as implemented in NetDraw 2.4 (Analytic Technologies, Lexington) (we manually repositioned a few nodes for clarity). In a Note at the end of this article, we describe procedures for identifying elicited partners uniquely. 


\section{Results}

\section{Index case characteristics}

There were no meaningful or statistically significant differences between seroconverters who participated in contact tracing and those who did not in terms of age, sex, race, education, employment, welfare, marital status, homelessness, recent incarceration, hepatitis $B$ vaccination, hepatitis $A$ vaccination, depression, behavioural sexual orientation, recent mental health treatment, current methadone treatment, age at first injection, recent needle exchange participation, or estimated numbers of recent injection partners/needle or syringe sharing partners/injection partners with whom other injection paraphernalia were shared. Twenty-two index participants seroconverted to $\mathrm{HCV}$ and four seroconverted to $\mathrm{HBV}$; one seroconverted to both HBV and HCV at the same assessment. Index cases included 20 men and six women, and their mean age was 31 years (median=30, range $=17-46$ ). Seventy-two percent of index cases were white, $12 \%$ were American Indian or Alaskan Native, and $16 \%$ belonged to another ethnic/racial group.

\section{Contact tracing outcomes}

Index cases reported a mean of 17 (median $=16$, range $=2-58$ ) injection partners. Twenty-three of the 26 index cases agreed to refer one or more partners. Of the 447 elicited partners, 160 (36\%) were sought for referral. (One index case was interviewed on the second to last day of the study, and therefore was not asked to refer his eight partners). Seventeen (10\%) referral vouchers, linked to nine index cases, were redeemed. Only eight of those vouchers were matched with confidence to a partner sought for referral by the corresponding index case, although two of these redeemers denied being current injectors. The available evidence suggested a further three persons redeeming vouchers were probably partners of the corresponding index case. Ten of 16 tested contacts were anti-HCV positive, and three of 14 were anti-HBc-positive. There were no meaningful differences in HCV or HBV prevalence between contacts matched to sought partners and those presenting vouchers who were not matched to sought partners. Some index cases spontaneously indicated why they would not refer partners. The reasons included the belief that most partners were already HCV infected ( $n=2$ index cases), court order to stay out of a drug area where most partners were located $(n=1)$, refusal to "hunt down" partners $(n=1)$, partner in inpatient drug treatment or hospital $(n=2)$, and a dying partner who posed no transmission risk $(n=1)$.

\section{Impact of supplementary elicitation techniques}

Index cases listed a mean of nine and median of eight injection partners before the supplementary elicitation techniques were administered. The Table shows that each supplementary elicitation technique elicited a noteworthy number and proportion of additional injection partners beyond those elicited in prior stages of the interview. Non-specific prompting and reading back the list each elicited additional injection partners from approximately half of the index cases. The additional partners reported in each of these stages represented $11-20 \%$ increases in the number of partners elicited on average. Most index cases listed additional injection partners in response to the location and network cues, and each set of these additional partners boosted the number elicited by 14 $29 \%$ on average. The supplementary techniques, in combination, elicited additional injection partners from $89 \%$ of index cases, and resulted in a mean of eight additional partners reported (essentially doubling the number elicited, on average).

The location cues were moderately more potent than the network cues. For the typical index case, 0.21 additional injection partners were elicited per location cue and 0.14 additional partners were elicited per network cue.

The number of partners index cases recalled before administration of the recall cues correlated positively with the number recalled in response to the location cues $(r=.45, p<.05)$ and network cues $(r=.26, p>.05)$. The number of freely recalled partners, however, was only weakly related to the number of additional partners elicited from nonspecific prompting $(r=.13, p>.05)$ and reading back the list $(r=.15, p>.05)$.

Eighteen index cases recalled partners both before the cues and by the cues; the two groups varied in locatability. Partners elicited by the cues tended to be somewhat less likely to be locatable or sought for referral (weighted mean $r=-0.27$, cumulative $Z=-4.3$, $\mathrm{p}<.001$, median $=-.20$, range $=-.82$ to $.68 ; 83 \%$ negative $).$ In

T A B L E

Effectiveness of the supplementary elicitation techniques

\section{F I G U R E}

Main component of injection network among index cases

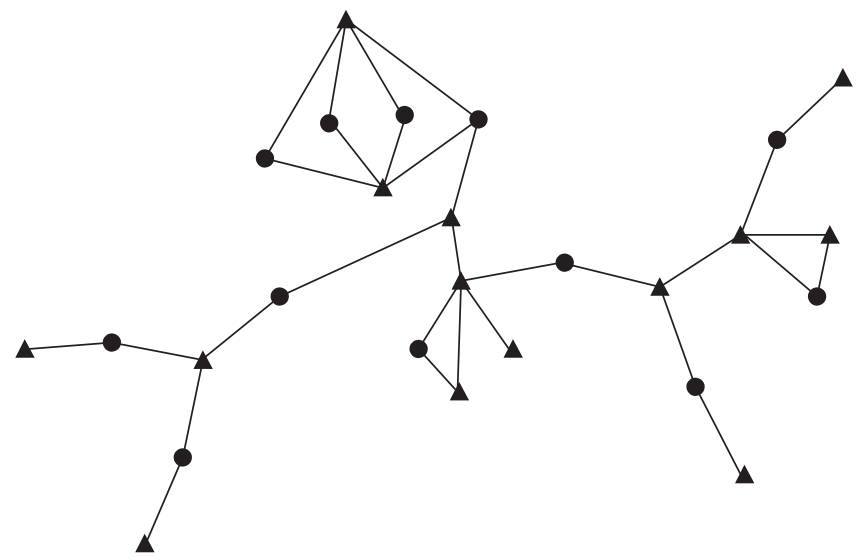

Triangles: index cases; circles: partners (who did not also participate as index cases). 
the aggregate, for all 26 index cases, injection partners elicited by the supplementary techniques were somewhat less likely to be locatable or to be sought for referral than those elicited during free recall (27\% versus $47 \%$ ).

\section{Description of injection network}

The Figure shows the main component (set of persons connected directly or indirectly by reported injection contact) of the injection network, based on reports from all 26 index cases. Index cases are represented as triangles, and partners who did not also participate as index cases are shown as circles. The main component includes 14 of the 26 index cases; other index cases were not linked to each other directly or indirectly, as far as we could determine. We excluded $76 \%$ of elicited partners from our analysis of the injection network because we could not identify them uniquely (the partners' first names were not rare, we judged their street nicknames not to be unique, or index cases referred to them with descriptions only). For clarity, partners mentioned by only one index case are not represented in the Figure, even if they had a rare first name or unique nickname.

Despite the severe restrictions on the data included for analysis, the measured network is fairly connected and cyclic (a cycle is a path involving more than two nodes that starts and ends at a particular node and, when it is traversed, each node is visited only once). However, index cases' recall periods were only $38 \%$ concurrent. That is, the sum of the pair-wise temporal overlap between index cases' date-specific recall periods was 38\% of the sum of the maximum possible overlap of index cases' recall periods (i.e. if all index cases had been interviewed on the same day). This means that some of the connectedness (and corresponding transmission potential) shown in the Figure might not have been present had the timing of partnerships been considered.

\section{Discussion}

Most IDUs who had recently seroconverted to HBV and/or HCV agreed to participate in patient referral contact tracing. Index cases reported many partners in contact interviews, and in the aggregate, index cases indicated they could refer more than one third of their elicited partners for testing. However, very few persons were ultimately referred and only half of them were confirmed as partners sought for referral. The supplementary elicitation techniques, and especially the recall cues, increased reporting of injection partners substantially. In all respects, our results mirrored prior evaluations of these techniques in other studies $[13,14]$, and the evidence of the techniques' effectiveness is now strong enough to make these techniques part of the standard interviewing practice for eliciting injection and sex partners [12]. Although partners elicited by the techniques were less likely to be locatable in the present study, technique-elicited partners are as likely as freely recalled partners to be infected with HCV [14]. The observed injection network was significantly connected and cyclic, as in previous studies of injection networks $[11,16,17]$. These structural characteristics are associated with epidemic spread of HBV, HCV, human immunodeficiency virus (HIV), and other sexually transmitted diseases [11,17-23]. The degree of connectivity and cyclicity we observed represents a minimum; with more complete data, network connectivity and cyclicity could only increase.

Our contact tracing procedures differed from conventional patient referral approaches in several ways that could have influenced the outcome. Index cases referred their partners for testing without notifying them of their specific exposure to HBV and/or HCV, although index cases were instructed to emphasise the high rate of infection among IDUs. Nonetheless, partners might not have appreciated the urgency for testing given this less personalised notification. Also, we paid index cases to participate in a contact interview. Many public health officials might not choose to make such payments for routine contact tracing, and it is unknown whether infected injectors would be willing to participate in contact tracing without such remuneration. Similarly, we paid referred partners for testing, and that might have increased the chance that a referred partner presented for testing. Furthermore, after completing the baseline interview in the cohort study, participants could refer other IDUs (regardless of whether they had injected together) to the cohort study. Referred persons were screened for eligibility and paid incentives, and index cases also received a "bounty" payment for each person successfully referred. Participants could make such referrals only at this initial point in the cohort study. We lacked sufficient resources in the contact tracing study to make payments to index cases for each partner referred. Index cases may have been less motivated to refer partners after having had the opportunity earlier to refer more easily located persons (any drug injectors) and earn additional money for doing so.

Despite these limitations, our contact tracing results are likely applicable to the investigation of any infectious disease in IDUs. To our knowledge, all prior successful contact tracing efforts with IDUs have been based on provider referral [17,24-29]. The spontaneous comments from some of our index cases about why they would not refer partners indicate that practical barriers to successful patient referral may be common among IDUs. Nonetheless, IDUs are willing to participate in contact tracing, but generally prefer that health workers locate and notify partners [25]. Our results suggest that provider referral is an essential ingredient of any contact tracing effort in IDUs. Moreover, highly connected injection networks imply that HBV/HCV transmission still might be controlled - to some extent - through contact tracing, even if some infected IDUs do not participate [30].

We identified index cases and reported partners as uniquely as possible by:

1. Partner nicknames mentioned multiple times that we judged to be uncommon(e.g. names similar to "Dragon", "Twist", and “Crocodile") and thus very likely to refer to the same person in this local setting;

2. Partner first names mentioned multiple times that were rare in the general population and thus very likely to refer to the same person $(<0.1$ persons expected to have a particular first name among the total number of partners mentioned by the index cases, as estimated from the Social Security Administration's first name database stratified by decade of birth (http:// www.ssa.gov/OACT/babynames/), weighting by the frequency of index cases by birth decade, and accounting for whether a name was used for females or males; birth decade weighting was based on decades of index cases' births, because Seattle IDUs tend to inject with similar age partners [11]);

3. Rare first names (by the same criterion) mentioned by index cases as partner names and which also were the names of other index cases.

By these criteria, the 16 reported partners included in analysis were uniquely identified by five nicknames, seven male first names, and four female first names. We performed simulations in which we randomly sampled first names from the Social Security Administration database with replacement for sample sizes equal to that of named partners in our study. Most simulation trials yielded no rare $(<0.1$ expected mentions) first names that were sampled two or more times, indicating our criterion was conservative (simulation details available on request).

\section{Aknowledgements}

We thank Eileen Hough for study coordination, Erica Seddig, Dawn Spellman, and Robert Yoon for data collection, Rong Lee for data management, Barbara Leigh and Peggy Peterson for overall project management, and John Roberts, Jr. for helpful discussions. This research was supported by National Institutes of Health grant no. R01DA015026. 


\section{References}

1. Toomey KE, Cates W Jr. Partner notification for the prevention of HIV infection. AIDS. 1989;3(Suppl 1):S57-S62.

2. Potterat JJ, Rothenberg R. The case-finding effectiveness of a self-referral system for gonorrhea: a preliminary report. Am J Public Health. 1977;67(2):174

3. Hagan H, Snyder N, Hough E, Yu T, McKeirnan S, Boase J, et al. Case-reporting of acute hepatitis B and C among injection drug users. J Urban Health. 2002;79(4):579-85.

4. Crofts N, Jolley D, Kaldor J, van Beek I, Wodak A. Epidemiology of hepatitis $C$ virus infection among injecting drug users in Australia. J Epidemiol Community Health. 1997;51(6):692-7.

5. Gunn RA, Weinberg MS, Borntrager D, Murray PJ. Partner notification for persons with chronic hepatitis B virus infection: use of a syphilis model service. Sex Transm Dis. 2006;33(7):437-40.

6. van Steenbergen JE, Baayen D, Peerbooms PG, Coutinho RA, Van Den Hoek A. Much gained by integrating contact tracing and vaccination in the hepatitis B antenatal screening program in Amsterdam, 1992-1999. J Hepatol. 2004:40(6):979-85.

7. Altice FL, Bruce RD, Walton MR, Buitrago MI. Adherence to hepatitis B virus vaccination at syringe exchange sites. J Urban Health. 2005;82(1):151-61.

8. Kuo I, Sherman SG, Thomas DL, Strathdee SA. Hepatitis B virus infection and vaccination among young injection and non-injection drug users: missed opportunities to prevent infection. Drug Alcohol Depend. 2004;73(1):69-78.

9. Ompad DC, Galea S, Wu Y, Fuller CM, Latka M, Koblin B, et al. Acceptance and completion of hepatitis B vaccination among drug users in New York City. Commun Dis Public Health. 2004;7(4):294-300.

10. Grebely J, deVlaming S, Duncan F, Viljoen M, Conway B. Current approaches to HCV infection in current and former injection drug users. J Addict Dis. 2008;27(2):25-35.

11. Brewer DD, Hagan H, Sullivan DG, Muth SQ, Hough ES, Feuerborn NA, et al. Social structural and behavioral underpinnings of hyperendemic Hepatitis $C$ virus transmission in drug injectors. J Infect Dis. 2006;194(6):764-72.

12. Brewer DD, Potterat JJ, Muth SQ, Malone PZ, Montoya PA, Green DA, et al. Randomized trial of supplementary interviewing techniques to enhance recall of sexual partners in contact interviews. Sex Transm Dis. 2005;32(3):189-93.

13. Brewer DD, Garrett SB. Evaluation of interviewing techniques to enhance recall of sexual and drug injection partners. Sex Transm Dis. 2001;28(11):666-77.

14. Brewer DD, Hagan H, Hough ES. Improved injection network ascertainment with supplementary elicitation techniques. Int J STD AIDS. 2008;19(3):188-91.

15. Rosenthal R. Meta-analytic procedures for social research. Newbury Park (CA): Sage Publications; 1991.

16. Rothenberg RB, Potterat JJ, Woodhouse DE, Muth SQ, Darrow WW, Klovdahl AS. Social network dynamics and HIV transmission. AIDS. 1998;12(12):1529-36.

17. Vogt TM, Perz JF, Van Houten CK Jr, Harrington R, Hansuld T, Bialek SR, et al. An outbreak of hepatitis B virus infection among methamphetamine injectors: the role of sharing injection drug equipment. Addiction. 2006;101(5):726-30.

18. Friedman SR, Neaigus A, Jose B, Curtis R, Goldstein M, Ildefonso G, et al. Sociometric risk networks and risk for HIV infection. Am J Public Health. 1997;87(8):1289-96.

19. Rothenberg RB, Sterk C, Toomey KE, Potterat JJ, Johnson D, Schrader M, et al. Using social network and ethnographic tools to evaluate syphilis transmission. Sex Transm Dis. 1998;25(3):154-60.

20. Potterat JJ, Phillips-Plummer L, Muth SQ, Rothenberg RB, Woodhouse DE, Maldonado-Long TS, et al. Risk network structure in the early epidemic phase of HIV transmission in Colorado Springs. Sex Transm Infect. 2002;78(Suppl 1):i159-63.

21. Potterat JJ, Muth SQ, Rothenberg RB, Zimmerman-Rogers H, Green DL, Taylor $\mathrm{JE}$, et al.. Sexual network structure as an indicator of epidemic phase. Sex Transm Infect. 2002;78(Suppl 1):i152-8.

22. Potterat JJ, Rothenberg RB, Muth SQ. Network structural dynamics and infectious disease propagation. Int J STD AIDS. 1999;10(3):182-5.

23. Rothenberg R, Kimbrough L, Lewis-Hardy R, Heath B, Williams OC, Tambe P, et al. Social network methods for endemic foci of syphilis. Sex Transm Dis. 2000;27(1):12-8.

24. Barata LC, Andriguetti MT, de Matos MR. Outbreak of malaria induced amon users of injectable drugs. Rev Saude Publica. 1993;27(1):9-14. [In Portuguese].

25. Levy JA, Fox SE. The outreach-assisted model of partner notification with IDUs. Public Health Rep. 1998;113(Suppl 1):160-9.

26. Munday PE, McDonald W, Murray-Sykes KM, Harris JRW. Contact tracing in hepatitis B infection. Br J Vener Dis. 1983;59(5):314-6.

27. Poulin C, Gyorkos TW, MacPhee J, Cann B, Bickerton J. Contact-tracing among injection drug users in a rural area. Can J Public Health. 1992;83(2):106-8.
28. Poulin $C$, Gyorkos $T$, Joseph $L$, Schlech $W$, 3rd, Lee $S$. An epidemic of hepatitis $B$ among injection drug users in a rural area. Can J Public Health. 1992;83(2):102-5.

29. Mercedes L, Heymans T, Saavedra J, Martimo J, Hooker C. Investigation of a cluster of acute hepatitis $\mathrm{C}$ virus (HCV) infections in injecting drug users (IDU), Minnesota, 2001. National STD Prevention Conference; 2002 March; San Diego, United States.

30. Potterat JJ, Muth JB, Murray C. Partner notification. Ann Intern Med. 1990;113(6):481.

This article was published on 9 April 2009

Citation style for this article: Brewer DD, Hagan $\mathrm{H}$. Evaluation of a patient referral contact tracing programme for hepatitis $\mathrm{B}$ and $\mathrm{C}$ virus infection in drug injectors. Euro Surveill. 2009:14(14):pii=19170. Available online: http://www.eurosurveillance.org/ ViewArticle.aspx?ArticleId $=19170$ 